\title{
Delivery of adjuvant chemotherapy (AC) to veterans with resected colon cancer.
}

\author{
Meeting: 2012 ASCO Annual Meeting \\ Category: Health Services Research
}

Subcategory: Quality and Quality Improvement (Including Outcomes and QOL Research)

Session Type and Session Title: Published in conjunction with the meeting.

Abstract Number: e16557

Citation: Riggs, H., Lane, K., Loehrer, P., Hui, S., Rawl, S., Ormerod, A., Weiner, M. (2012).

Delivery of adjuvant chemotherapy to veterans with resected colon cancer. [Abstract published in the Proceedings of the 2012 ASCO Annual Meeting, June, 2012.] J Clin Oncol 30, 2012. (Suppl; abstr e16557).

Author(s): Heather Dawn Riggs, Kathleen A. Lane, Patrick J. Loehrer, Siu Hui, Susan Rawl, Anne Ormerod, Michael Weiner; Indiana University Melvin and Bren Simon Cancer Center, Indianapolis, IN; Indiana University School of Medicine, Indianapolis, IN; Indiana University School of Nursing, Indianapolis, IN; Indiana University Health, Indianapolis, IN; VHA HSR\&D HFP 04-148 at Richard L. Roudebush VA Medical Center, Center of Excellence on Implementing Evidence-Based Practice, Indianapolis, IN

Background: Patients (pts) with incomplete administration of AC for colon cancer (CC) likely experience inferior survival while oxaliplatin- and capecitabine-containing AC may not improve survival for older pts. The influence on AC completion of age, other baseline patient characteristics, or regimen characteristics is unknown. We evaluated the association of baseline characteristics and planned AC regimen with AC delivery in resected CC pts.

Methods: This retrospective cohort study identified stage II and III resected CC pts from our local VA Medical Center tumor registry who initiated AC between 2004 and 2010. AC delivery was measured as percentage of planned cumulative dose (PCD) of 5-fluorouracil or capecitabine delivered. Baseline characteristics were demographics, CC stage, travel distance to the treatment site (TD), Adult Comorbidity Evaluation-27 score, and labs. We classified AC as fluoropyrimidine (oral or IV)-based and as oxaliplatin-containing. Linear regression tested each association with PCD. We recorded occurrence of toxicity and death. Cox regression models were used for survival analysis.

Results: Fifty pts (98\% male, median age 63) initiated AC for stage II ( $n=6)$ or III ( $n=44)$ disease with median interval from resection to AC of 7.4 weeks. The median PCD was 75\%; median treatment duration was 5 months. Lower PCD was associated with older age $(p=0.05)$, lower GFR ( $\mathrm{p}=0.05)$, higher TD ( $\mathrm{p}=0.02)$, lower baseline hemoglobin $(\mathrm{p}=0.10)$, and receipt of capecitabine $(\mathrm{p}<0.01)$. Receipt of capecitabine $(\mathrm{p}=0.008)$ and TD $(\mathrm{p}=0.04)$ remained significantly associated with PCD in a multiple linear regression model. Toxicity was noted for $78 \%$, and PCD tended to be lower for reported cardiac events $(p=0.07)$. Pts older than 63 had 
inferior survival $(\mathrm{HR}=4.17, \mathrm{p}=0.03)$. Pts receiving less than $80 \% \mathrm{PCD}$ tended toward inferior survival $(\mathrm{HR}=4.31, \mathrm{p}=0.07)$.

Conclusions: Capecitabine-based AC and travel distance were associated with lower PCD, which may negatively impact survival. Efficacy of shorter courses of oxaliplatin-based chemotherapy is being evaluated elsewhere. Close attention to quality prescribing and monitoring practices for capecitabine are recommended. These findings should be confirmed in a larger cohort. 